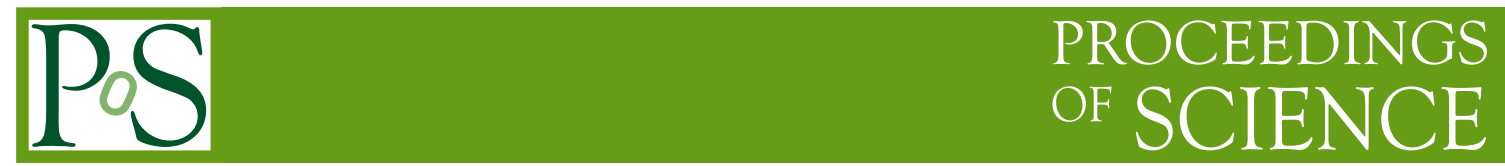

\title{
Width of the Confining String
}

\author{
F.V. Gubarev*t \\ Institute of Theoretical and Experimental Physics, \\ B. Cheremushkinskaya 25, Moscow, 117218, Russia \\ E-mail: gubareveitep.ru
}

We critically reconsider our recent observation of confining string shrinkage in pure glue SU(2) lattice gauge theory near the continuum limit. Using the advanced numerical techniques we argue that imperfect overlap with the string ground state and corresponding ambiguities in $T \rightarrow \infty$ extrapolation are likely to be the cause of the observed scaling violations. In particular, even in the limit of large Euclidean times the string shrinkage is apparent in torelon correlation function, however, in this case the best relevant overlap we could attain is $\sim 50 \%$. To the contrary, when the ground state is selected properly the string width scales in physical units being $\approx 0.3 \mathrm{fm}$ for $1 \mathrm{fm}$ long confining string.

8th Conference Quark Confinement and the Hadron Spectrum

September 1-62008

Mainz, Germany

\footnotetext{
* Speaker.

${ }^{\dagger}$ Work was supported by RFBR-07-02-00237-a, RFBR-08-02-00661-a grants, computations had been partially performed at Joint Supercomputer Center, RAS.
} 


\section{Introduction}

Formation of the confining string between external heavy quark and antiquark naturally explains the linearly rising heavy quark potential as well as specific stringy corrections present in it [1]. This phenomenon is confirmed in numerous lattice studies (see, e.g., Refs. [2]), which however are always limited to some finite range of UV cutoff (lattice spacing $a$ ). However, the internal structure of the confining string in terms of various gluonic observables is less known, although it is generically expected and confirmed on the lattice $[2,3]$ that within the flux tube the energy density is enhanced, while the action density, the magnitude of topological fluctuations and other quantities are suppressed with respect to the corresponding vacuum expectations. Note that the local action density difference

$$
\Delta s=\langle s\rangle_{0}-\langle s\rangle_{q \bar{q}}>0, \quad s=\operatorname{Tr} F_{\mu v}^{2}
$$

is distinguished since its integral matches the heavy quark potential [4]. In the limit of large $q \bar{q}$ distances $R$ the corresponding relation for $\mathrm{SU}(2)$ gauge group reads

$$
\int d^{2} r \Delta s=2 \sigma \beta_{f}, \quad \beta_{f}=-\frac{\partial \ln a}{\partial \beta} \stackrel{a \rightarrow 0}{\longrightarrow} \frac{6 \pi^{2}}{22},
$$

where the integral is over the flux tube transverse cross section and $\sigma$ is the string tension. Note that the rigorous sum rule (1.2) says hardly anything about actual action density profile. Nevertheless, one expects rather generically that its functional form is to be Gaussian [5]

$$
\Delta s(r, R)=C(R) \exp \left\{-r^{2} / \delta^{2}(R)\right\}, \quad \delta^{2}(R)=\frac{\zeta}{\pi \sigma} \ln \left[R / R_{0}\right],
$$

where we quoted common to all effective string theories prediction for the squared width $\delta^{2}$ of the flux tube, $\zeta$ is model-dependent numerical constant and $R_{0}$ is UV cutoff of the effective theory. True that the string action density profile attracted much attention in the past [2]. The summary of these investigations is that both the Gaussian shape and the string widening, Eq. (1.3), are qualitatively confirmed for large enough quark-antiquark separations $R \gtrsim 0.7 \mathrm{fm}$. Although quantitatively there are points worth to be improved, the above qualitative picture will hardly change. Thus the question is why we decided to consider the flux tube profile again and what are the subtle points we hope to shed some light on?

Briefly, the reason reduces to the recent observation that non-perturbative fluctuations, commonly considered in relation to confinement and known as Abelian monopoles and center vortices, have a non-trivial ultraviolet structure [6] (see [7, 8] for comprehensive discussions). Namely, the excess of non-Abelian action associated with both the monopoles and vortices is UV divergent, while their number density perfectly scales towards the continuum limit. On the other hand, in Yang-Mills theory all UV divergences must be interpretable within the field theory itself. Confronting the field theory with the lattice data one concludes that vacuum defects (monopoles and vortices) are, in a sense, dual to high orders of perturbation theory (see [7] for details). For the present discussion it is crucial that for the action density the impact of the vacuum defects (or, alternatively, of the ambiguities of the perturbation theory at high orders) is to be parametrized by an additional quadratic term in the conventional OPE-based expansion

$$
\langle s\rangle_{0}=a^{-4} \cdot\left\{\sum_{n=0}^{N} b_{n} \alpha_{s}^{n}+c_{2}^{N}\left(a \cdot \Lambda_{Q C D}\right)^{2}+c_{4}\left(a \cdot \Lambda_{Q C D}\right)^{4}\right\} .
$$


The necessity of the quadratic correction is quite evident in the context of vacuum defects. However, from the field-theoretical viewpoint, which is actually implied by (1.4), a remark have to be added. Namely, Eq. (1.4) explicitly refers to a particular N-loop order of perturbative expansion, the divergent reminder of which is parametrized by the second term. In this respect (1.4) does not follow from the theory itself, rather it reflects the inability to handle high orders of $\alpha_{s}$-expansion and the successful phenomenology of quadratic power corrections (see Ref. [9] and references therein). In particular, by no means Eq. (1.4) implies OPE violation. Consequently, in the field theory settings the quadratic correction cannot be discussed out of context of the perturbation theory. This is reflected in the explicit $N$-dependence of the coefficient $c_{2}^{N}$ : with increasing number of perturbative loops explicitly accounted for it drops rapidly being (numerically) compatible with zero in the empirical limit $N \rightarrow \infty$ [10]. Nevertheless, for moderate $N$, when the quadratic term could be detected, it turns out to be parametrically small [11]

$$
c_{2}^{N} \Lambda_{Q C D}^{2} \lesssim[50 \mathrm{MeV}]^{2},
$$

which is only an order of magnitude estimation (concrete numbers vary depending on the details of the measurements).

Given the above uncertain status of the quadratic divergence it is legitimate to ask whether the issue is at all relevant. The fair answer seems to be affirmative because we aware of the significance of vacuum defects to QCD vacuum structure. The discovery of non-trivial UV structure of monopoles/vortices provides a bridge to the field theory linking them to the question of power divergences. Hence it is worth to consider Eq. (1.4) in its generality and to consider the most general power dependence of $\langle s\rangle_{0}$ upon the UV/IR scales

$$
\langle s\rangle_{0}=c_{0}^{(0)} \cdot a^{-4}+c_{2}^{(0)} \Lambda_{Q C D}^{2} \cdot a^{-2}+c_{4}^{(0)} \Lambda_{Q C D}^{4}
$$

with some unknown coefficients. True that Eq. (1.6) by itself makes no much sense because the vacuum action density is not observable anyway. However, it is crucial that once the quadratic term does not vanish identically no symmetry could ever prevent its dependence upon the external soft fields. Hence the corresponding VEV $\langle s\rangle_{q \bar{q}}$ is to be parametrized analogously with a priori distinct coefficients $c_{2,4}^{(q \bar{q})}$. Then the difference (1.1) inside the string is given by

$$
\Delta s=c_{2} \Lambda_{Q C D}^{2} \cdot a^{-2}+c_{4} \Lambda_{Q C D}^{4},
$$

while Eq. (1.5) provides an upper bound on $c_{2} \Lambda_{Q C D}^{2}$. It is worth mentioning that in terms of vacuum defects Eq. (1.7) with $c_{2} \neq 0$ follows directly from the known distribution of monopoles [12] and vortices [13] in the vicinity of the flux tube. Combining (1.2), (1.7) one concludes that nonvanishing $c_{2}$ coefficient manifests itself in peculiar linear shrinkage of the flux tube width with simultaneous quadratic divergence of the height of the Gaussian profile (1.3)

$$
\delta^{2} \sim \sigma a^{2} / \Lambda_{Q C D}^{2}, \quad C(R) \sim \Lambda_{Q C D}^{2} \cdot a^{-2}
$$

in the limit $a \rightarrow 0$ (insisting on Gaussian profile seems to be safe at any finite $a$ ). Note that this behavior is only asymptotic, an estimation of the relevant lattice spacings below which (1.8) settles in could be obtained from (1.5) and by noting that the term $c_{4} \Lambda_{Q C D}^{4}$ is to be of order gluon condensate. 


\begin{tabular}{c|c|c|c|c|c}
\multirow{2}{*}{$\beta$} & \multirow{2}{*}{$a, \mathrm{fm}$} & $V_{\text {lat }}, V_{\text {phys }}$ & \multicolumn{2}{|c|}{ Multilevel: $\Delta$ and $N_{\text {upd }}$} & Multichannel, \\
& & & Torelons, $N_{\text {lev }}=2$ & Wilson loops, $N_{\text {lev }}=3$ & $N_{\text {smearing }}$ \\
\hline 2.5046 & 0.081 & $28^{4},[2.3 \mathrm{fm}]^{4}$ & $(14),(4000)$ & - & - \\
\hline 2.5515 & 0.072 & $28^{4},[2.0 \mathrm{fm}]^{4}$ & $(14),(3500)$ & - & - \\
& & $32^{4},[2.3 \mathrm{fm}]^{4}$ & $(16),(5000)$ & $(4,8),(12,300)$ & $(50,65,80)$ \\
\hline 2.6800 & 0.047 & $36^{4},[1.7 \mathrm{fm}]^{4}$ & $(18),(4500)$ & - & - \\
& & $40^{4},[1.9 \mathrm{fm}]^{4}$ & $(20),(5000)$ & $(5,10),(12,600)$ & $(150,180,210)$ \\
\hline 2.7600 & 0.034 & $48^{4},[1.8 \mathrm{fm}]^{4}$ & $(24),(5000)$ & $(6,12),(12,1200)$ & $(180,210,240)$ \\
\hline
\end{tabular}

Table 1: Ensembles used in our study. Multilevel notations (fourth column) closely follow [18]. APE smearing (fifth column) had been performed with $\varepsilon$ parameter 0.25 .

Straightforward calculation shows that the divergence (1.8) should be manifest for $a \lesssim 0.03 \mathrm{fm}$ and signifies that numerically the status of quadratic divergence is also uncertain. Indeed, the relevant scale is rather small parametrically and still leaves slight possibility that the divergence (1.7) could have been missed in the previous studies. The prime purpose of our investigations, which we summarize in this paper, is to study the scaling properties of the action density distribution within the long confining string. For technical reasons we restricted ourselves to SU(2) pure gauge theory with Wilson action, summary of simulation parameters is given in Table 1.

Our first measurements [14] confirmed the presence of the quadratic term, Eq. (1.7), with magnitude being in agreement with (1.5). However, we had never been quite satisfied with this result for the following reasons:

1. Ground state separation (ground state overlap).

String creation/annihilation operators used in [14] were not optimal, the relevant overlaps with the ground state of heavy $q \bar{q}$-pair were of order $80 \div 90 \%$. This required us to make rather complicated fit to $T \rightarrow \infty$ thus introducing systematic biases the magnitude of which we were unable to fairly estimate. This issue is addressed in Section 3.

2. Limit of large $q \bar{q}$ separations.

Ultimately, we're interested in the physics of long confining string, $R \gg R_{0} \approx 0.7 \mathrm{fm}$, when the $q \bar{q}$ separation $R$ greatly exceeds the string formation length $R_{0}$. With Wilson loops (or Polyakov lines) it is hardly possible to go far beyond $R_{0}$ due to the technical limitations, hence the string profile is disturbed by singular point-like sources at its end-points. Again some phenomenology had been applied in [14] leading to the same question of systematic errors. Ideally, the point-like sources have to be removed, which leads us to consider the torelon correlation function (Section 2).

An overall conclusion of the present study is that it is the overlap problem which is the most crucial to get the physics right. In particular, when the proper selection of string ground state is ensured and its $T=\infty$ asymptotic structure is directly seen the effect of string shrinkage disappears. To our surprise the string profile turned to be extremely sensitive in this respect, contrary to, e.g., heavy quark potential in which we never observed scaling violations. Therefore in string profile studies it is absolutely necessary to get rid of excited states contamination prior to making any conclusions on the flux tube internal structure. 

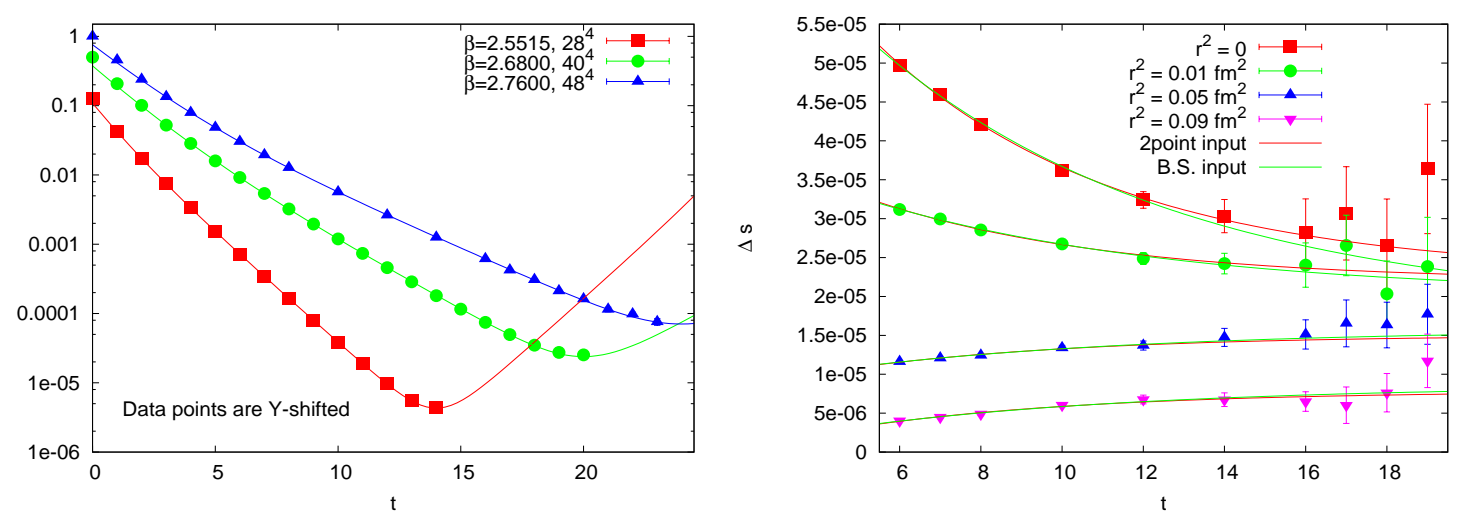

Figure 1: Left: torelon correlator at various spacings/volumes and two-exponential fits $\mathscr{T}(t)=\sum c_{i} e^{-m_{i} t}$, $c_{0} \approx 0.4, c_{1} \approx 0.55$. Right: examples of $t \rightarrow \infty$ fits with gap fixed either from $\mathscr{T}(t)$ or from bosonic string theory (B.S.), $\beta=2.760,48^{4}$.

\section{Torelons}

Technically, the torelon operator is similar to Polyakov line: it is the gauge holonomy around the spatial period of toroidal lattice in a particular time slice (see, e.g., Ref. [15] and references therein). Physically, it is quite different however: torelon creates confining string closed through the periodic boundary conditions and stable for topological reasons. The string exists without pointlike sources, hence the torelon correlation function is best suited to study the flux tube internals. Normalized torelon correlator allows usual spectral decomposition $\mathscr{T}(t)=\sum c_{i} e^{-m_{i} t}, \mathscr{T}(0)=1$, where masses $m_{i}$ depend upon the spatial lattice extent $L$ and have the same leading asymptotic $m_{i} \approx \sigma L$.

In order to study the string profile with torelons it is necessary to have precise data for the torelon correlation function at large Euclidean times. This is due to the local action density insertion: for instance, we used clover-like sum of all neighboring to given point plaquettes, hence the minimal Euclidean time we could consider is $t=4$ (in fact, it has to be larger for asymptotic exponential falloff to take place). Thus the use of modern multilevel technique [16, 17] is mandatory. In usual multilevel approaches it is the time axis which is divided into slabs, so that at large Euclidean times torelon creation/annihilation operators belong to different time slabs. Our experience showed, however, that in order to obtain desired precision in string profile, the 2-point correlator should be known with by order of magnitude better accuracy than it is available with usual scheme. Therefore, we utilized another approach in which the torelon direction is divided among different slabs. The ground state enhancement is done as usual in each slab, however, it is evident that our scheme does not allow to get optimal overlap with the string ground state: the more slabs are introduced the worse is overlap. Indeed, in each slab one considers straight smeared spatial holonomies with end-points fixed at the boundaries. Upon averaging the string wavefunction is constructed as superposition of various paths, which are required, however, to pierce prescribed boundary points. Effectively at each boundary one introduces a node of the wavefunction thus reducing the ground state overlap. Minimal number of time slabs with the 2-level scheme is two and the corresponding overlap is about $50 \%$. Despite of its smallness we hoped to eliminate exited states contamination 

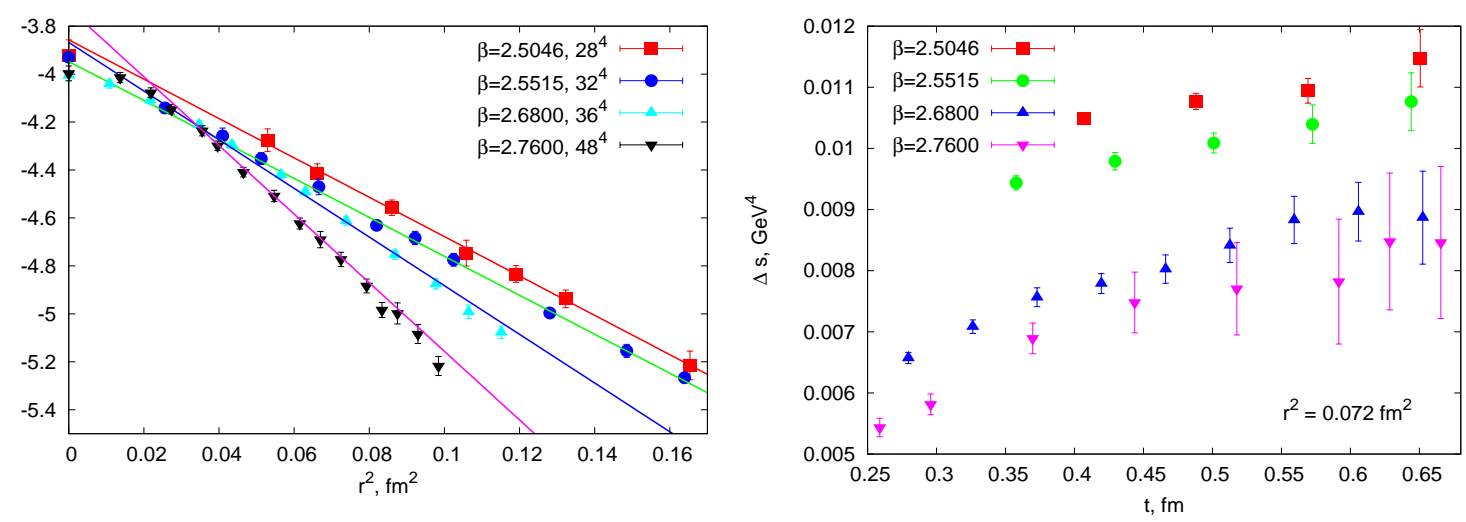

Figure 2: Left: $\ln \left(\Delta s(t=\infty) /[1 \mathrm{GeV}]^{4}\right)$ vs. $r^{2}$ at various lattice resolutions obtained with fixed gap fits (points) and cumulative one (solid lines). Right: $\Delta s(t)$ at $r \approx 0.27 \mathrm{fm}$ at different lattice spacings.

by attaining the limit of large Euclidean times, which is feasible with multilevel strategy.

Sample of data points obtained for the torelon correlator is shown on Fig. 1 (left). Analyzes reveal that we see a superposition of two states with approximately equal amplitudes. Mass extraction is done via two-exponential fit, lower $t$-limit of which was varied until lighter mass stabilizes. Masses extracted this way are in qualitative agreement with the literature [15], however, quantitatively they appear to be systematically slightly larger. This is because we are obliged to consider 2-point correlator, not the wall-wall correlation function, and hence inevitably get an admixture of non-zero lowest spatial momentum states. Thus the masses extracted from 2-point function could only be used as an approximation to the true spectral gap in the torelon channel.

Unfortunately, our hope to observe exclusively the ground state profile at largest available times failed for small transverse distances (Fig. 1, right). Indeed, for $r^{2} \lesssim 0.03 \mathrm{fm}^{2} t$-dependence of $\Delta s\left(t, r^{2}\right)$ is apparent, while at larger $r^{2}$ an approximate $t$-constancy of the profile seems to settle at $t \gtrsim 0.5 \mathrm{fm}$. Therefore, it is necessary to make $t \rightarrow \infty$ extrapolation for which the leading finite- $t$ correction looks like single exponent with gap being fixed in term of the spectral gap in the torelon channel. However, besides the imprecise knowledge of the gap there is an additional limitation: due to imperfect overlap with the ground state single exponent might not be adequate especially at small $r$. In view of these shortcomings various fits were tried out: 1 . Single exponent fits with gap fixed either from the 2-point function or from the bosonic string theory prediction (they are illustrated Fig. 1, right). 2. Fit by constant in $t$-range where data points come to plateau, it was used for qualitative purposes only. 3. Cumulative fit to all available $t, r^{2}$ points with single exponential ansatz and treating the gap as free parameter. To ensure the validity of single exponent approximation the points too close to the string axis were excluded, the corresponding cut on $r$ is obtained from previous fits. Additionally, we made seemingly well grounded assumption that the string profile at $t=\infty$ is to be Gaussian. This greatly reduces the dimensionality of the problem and allows much better precision.

Summary of the results obtained with various fitting procedures is presented on Fig. 2, left panel. Briefly, all the above fits appear to be adequate and consistent with each other except for the region $r^{2} \lesssim 0.03 \mathrm{fm}^{2}$ at smallest lattice spacing, where fixed gap fits notably deviate from Gaussian. Treating the discrepancies at small $r$ as a consequence of the imperfect overlap and the enforcement 

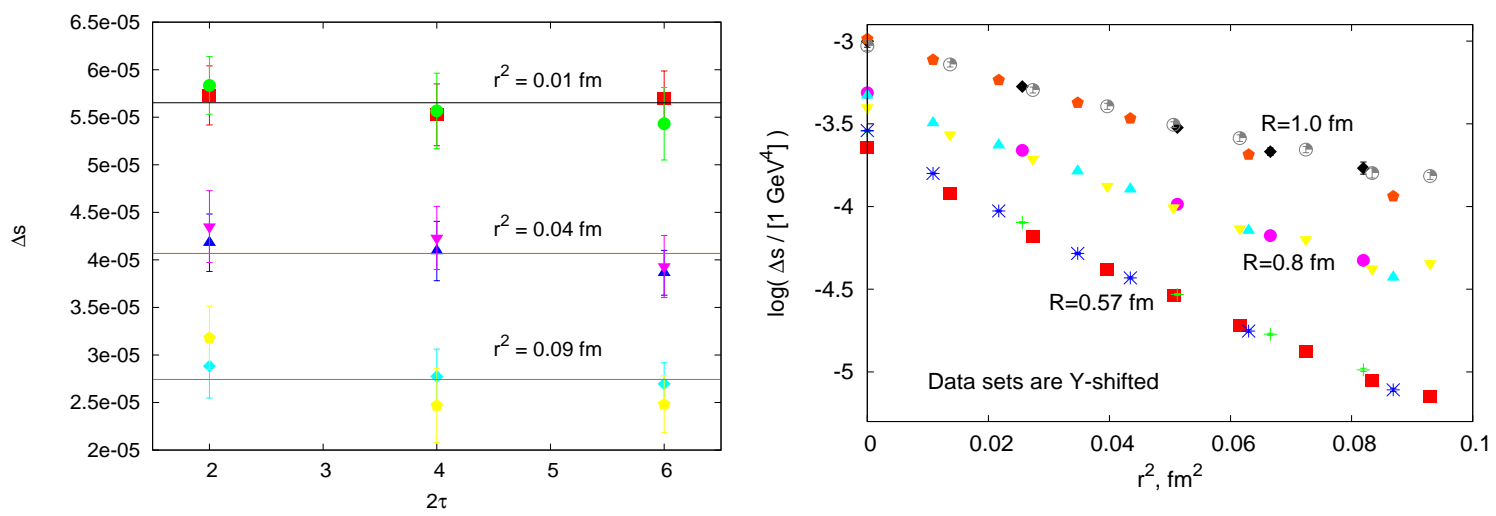

Figure 3: Left: $\Delta s$ vs. $\tau$ at $\left(t_{0}, t\right)=\{(8,18),(8,22)\}, R=0.93 \mathrm{fm}$ and different $r^{2}\left(\beta=2.680,40^{4}\right)$ with the corresponding fits to constant. Right: flux tube profiles at various lattice resolutions.

of single exponential anzatz, we conclude that regardless of actual $t \rightarrow \infty$ extrapolation the string width seems to drop with diminishing lattice spacing. In fact, the apparent string shrinkage could be identified directly by looking on the data at roughly the same transverse distance $r \approx 0.27 \mathrm{fm}$ and various lattice resolutions (Fig. 2, right). Furthermore, the magnitude of the quadratic divergence turns out to be $c_{2} \Lambda_{Q C D}^{2} \approx[30 \mathrm{MeV}]^{2}$ and essentially the same number follows from the consideration of the string profile height. Although the above treatment seems to be self-consistent, it is crucial to investigate the systematic biases of the ground state extraction, to which we turn next.

\section{Wilson loops}

It seems that the only reliable way to estimate the precision of $t \rightarrow \infty$ extrapolation is to get rid of it and to ensure that only the ground state propagates in the considered channel. To this end we combined the multilevel (actually, 3-level) scheme of Wilson loops calculation with the special ground state selection technique conventionally known as multichannel [19]. In essence it reduces to choosing the set $\{|i\rangle\}, i=1, \ldots, N_{s t}$ of string states and solving the generalized eigenvalue problem (GEVP)

$$
W_{i j}(t, R) e_{j}^{(\lambda)}=\lambda W_{i j}\left(t_{0}, R\right) e_{j}^{(\lambda)} .
$$

Here $W_{i j}(t, R)$ is $t \times R$ Wilson loop correlator evaluated for the states $|i\rangle,|j\rangle$, which we prepared using different number of APE smearing sweeps with the same smearing parameter (see Table1). The best available approximation to the ground state corresponds to the eigenvector $e^{(0)}$ with maximal eigenvalue $\lambda^{(0)}$. For the string profile studies this approach applies literally and leads to

$$
\Delta s=\langle s\rangle_{0}-e_{i}^{(0)}[W s]_{i j} e_{j}^{(0)} / \lambda^{(0)},
$$

where $[W s]_{i j}$ correlation matrix is similar to $W_{i j}$, but with local action density operator inserted at time-like [transverse] distances $\tau$ [ $r$ from the loop geometrical center (longitudinal shift was kept vanishing). Technically, our approach is similar to that of Ref. [17]: at the lowest (deconfining) level of our 3-level scheme only the products of time-like open holonomies (possibly with action density insertions) were averaged. At the next (confining) level these were accumulated and open 

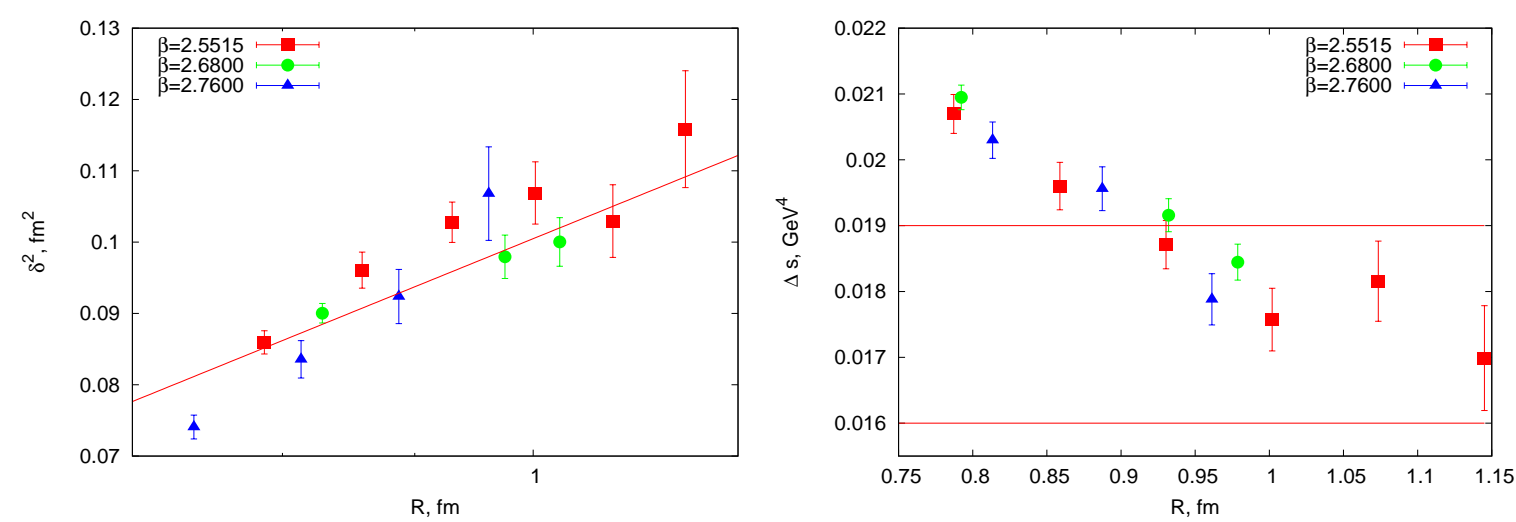

Figure 4: Left: Squared string width $\delta^{2}$ versus distance $R$ plotted in logarithmic scale. Straight line illustrates the bosonic string theory prediction (1.3), $\zeta=1$, with fitted parameter $R_{0} \approx 0.2 \mathrm{fm}$. Right: height of the Gaussian profile, $\Delta s\left(r^{2}=0\right)$, as a function of $R$, solid lines represent the rough upper/lower estimates of the gluon condensate.

staple-like segments of Wilson loops were calculated as well (to reduce the statistical errors the space-like pieces of the loops were required to be at least $l_{c r} / 2$ distance away from the slab boundaries, $l_{c r}$ being the inverse temperature of deconfinement transition at fixed boundaries). Finally, at the upper-most level the averaged open holonomies and their tensor products were combined to obtain the correlation matrices $W_{i j}$, $[W s]_{i j}$. Note that with definition (3.2) $\Delta s$ depends upon five parameters, $\left(t_{0}, t, \tau, R, r^{2}\right)$, where the triple $\left(t_{0}, t, \tau\right)$ is used exclusively to ensure the proper ground state selection.

The multichannel approach with three trial states allows to significantly increase the ground state overlap. In all cases it was at least 0.99 differing from unity in the third digit well within the statistical errors. As a consequence the dependence $\Delta s\left(t_{0}, t, \tau\right)$ tuned to be trivial as is exemplified for $\beta=2.680,40^{4}$ data on Fig. 3 (left), where $\Delta s$ at $R=0.93 \mathrm{fm}$ is plotted as a function of $\tau$ with two choices of GEVP window $\left(t_{0}, t\right)=\{(8,18),(8,22)\}$ and various transverse distances. The straightforward constant fit gives then the $t=\infty$ asymptotic of the string profile, which is presented on Fig. 3, right. To our surprise, the points obtained at various lattice resolutions fall, in fact, on the top of each other with no sign of scaling violations. The only conclusion we could draw is that the undertaken $t \rightarrow \infty$ extrapolations are in fact ambiguous despite of their apparent self-consistency. Therefore the previous claim of the string shrinkage is void and at least within the precision of our data the quadratic divergence is not seen in the action density difference (1.1).

Once the claim of linear vanishing of the string width with the lattice spacing had been abandoned it is worth to consider the dependence of $\delta^{2}$ upon the distance $R$ (Fig. 4, left). According to (1.3) it is expected to be logarithmically increasing function and indeed Fig. 4 qualitatively confirms this. It is also apparent that the data does not allow quantitative conclusions, only order of magnitude estimates could be given. To this end we tried the logarithmic fit of the type (1.3). Although the data prefer $\zeta$ value slightly above one, the difference is insignificant. Thus we fixed $\zeta$ to unity and evaluated the $R_{0}$ parameter, the optimal value of which turned to be $R_{0} \approx 0.2 \mathrm{fm}$.

Finally, let us remark that for $\approx 1 \mathrm{fm}$ long confining string the height of its action density profile is about $0.018 \mathrm{GeV}^{4}$ (Fig. 4, right). Numerically, this is very similar to the magnitude of 
the gluon condensate in SU(2) gluodynamics [20]. We qualitatively conclude therefore that gluon condensate vanishes on the symmetry axis of well developed confining string.

\section{Conclusions}

In this paper we critically reviewed the observation of the confining string shrinkage in the continuum limit of pure glue SU(2) lattice Yang-Mills theory. The aim was to address the problem of systematic biases coming from the imperfect ground state determination and from the string profile distortions due to the presence of singular point-like sources at its end-points. The second question is proved to be inessential: in the case of torelon correlator, where the flux tube exists without point-like sources, the string shrinkage had been also identified. However, addressing the issue of excited states contamination we discovered that it is a very delicate and perhaps not a well defined problem of taking the limit of infinite Euclidean times. Despite of apparent self-consistency of considered $t \rightarrow \infty$ extrapolations they lead to incorrect results. Namely, in the case of Wilson loops it is possible to construct almost perfect approximation to the string ground state, the shape of which is in fact independent upon the Euclidean time extent. Then the effect of string shrinkage completely disappears proving that it is an artifact of improper ground state selection. As a byproduct of our analyzes we qualitatively confirm the validity of the effective bosonic string theory description of the string profile and argue that the gluon condensate vanishes within the well developed confining flux tube.

\section{References}

[1] M. Luscher and P. Weisz, JHEP 0207, 049 (2002) [arXiv:hep-lat/0207003]. M. Luscher and P. Weisz, JHEP 0407, 014 (2004) [arXiv:hep-th/0406205].

N. D. Hari Dass and P. Majumdar, JHEP 0610, 020 (2006) [arXiv:hep-lat/0608024].

N. D. Hari Dass and P. Majumdar, PoS LAT2005, 312 (2006) [arXiv:hep-lat/0511055].

K. J. Juge, J. Kuti and C. Morningstar, Phys. Rev. Lett. 90, 161601 (2003) [arXiv:hep-lat/0207004].

[2] G. S. Bali, K. Schilling and C. Schlichter, Phys. Rev. D 51, 5165 (1995) [arXiv:hep-lat/9409005].

P. Pennanen, A. M. Green and C. Michael, Phys. Rev. D 56, 3903 (1997) [arXiv:hep-lat/9705033]. M. Fukugita and T. Niuya, Phys. Lett. B 132, 374 (1983).

A. M. Green, C. Michael and P. S. Spencer, Phys. Rev. D 55, 1216 (1997) [arXiv:hep-lat/9610011].

R. W. Haymaker et al. Phys. Rev. D 53, 389 (1996) [arXiv:hep-lat/9406021].

F. Okiharu and R. M. Woloshyn, Nucl. Phys. Proc. Suppl. 129, 745 (2004) [arXiv:hep-lat/0310007].

F. Bissey et al. Phys. Rev. D 76, 114512 (2007) [arXiv:hep-lat/0606016].

J. Wosiek and R. W. Haymaker, Phys. Rev. D 36, 3297 (1987).

[3] V. G. Bornyakov et al., Phys. Rev. D 70, 054506 (2004) [arXiv:hep-lat/0401026].

M. N. Chernodub and F. V. Gubarev, Phys. Rev. D 76, 016003 (2007) [arXiv:hep-lat/0703007].

[4] F. Karsch, Nucl. Phys. B 205, 285 (1982).

C. Michael, Nucl. Phys. B 280, 13 (1987).

H. J. Rothe, Phys. Lett. B 355, 260 (1995) [arXiv:hep-lat/9504012].

[5] M. Luscher, G. Munster and P. Weisz, Nucl. Phys. B 180, 1 (1981).

M. Luscher, Nucl. Phys. B 180, 317 (1981).

[6] V. G. Bornyakov et al. Phys. Lett. B 537, 291 (2002) [arXiv:hep-lat/0103032].

F. V. Gubarev et al. Phys. Lett. B 574, 136 (2003) [arXiv:hep-lat/0212003].

F. V. Gubarev et al. Nucl. Phys. Proc. Suppl. 129, 671 (2004) [arXiv:hep-lat/0309034]. 
[7] V. I. Zakharov, arXiv:hep-ph/0309178. V. I. Zakharov, arXiv:hep-ph/9812374.

[8] V. I. Zakharov, arXiv:hep-ph/0309301.

V. I. Zakharov, Phys. Atom. Nucl. 68, 573 (2005) [Yad. Fiz. 68, 603 (2005)] [arXiv:hep-ph/0410034].

V. I. Zakharov, Phys. Usp. 47 (2004) 37 [Usp. Fiz. Nauk 47 (2004) 39].

V. I. Zakharov, Nucl. Phys. Proc. Suppl. 164, 240 (2007) [arXiv:hep-ph/0509114].

[9] F. V. Gubarev, M. I. Polikarpov and V. I. Zakharov, Surveys High Energ. Phys. 15, 89 (2000).

[10] R. Horsley et al. Nucl. Phys. Proc. Suppl. 106, 870 (2002) [arXiv:hep-lat/0110210].

P. E. L. Rakow, PoS LAT2005, 284 (2006) [arXiv:hep-lat/0510046].

[11] G. Burgio et al. Phys. Lett. B 422, 219 (1998) [arXiv:hep-ph/9706209].

F. Di Renzo and L. Scorzato, JHEP 0110, 038 (2001) [arXiv:hep-lat/0011067].

P. Y. Boyko, F. V. Gubarev, S. M. Morozov, Phys. Rev. D 73, 014512 (2006) [arXiv:hep-lat/0511050].

[12] T. Suzuki, Nucl. Phys. Proc. Suppl. 30, 176 (1993).

G. S. Bali et al. Prog. Theor. Phys. Suppl. 131, 645 (1998) [arXiv:hep-lat/9802005].

G. S. Bali et al. Nucl. Phys. Proc. Suppl. 63, 519 (1998) [arXiv:hep-lat/9709114].

R. W. Haymaker, Phys. Rept. 315, 153 (1999) [arXiv:hep-lat/9809094].

F. V. Gubarev et al. Phys. Lett. B 468, 134 (1999) [arXiv:hep-lat/9909099].

[13] V. G. Bornyakov et al. JETP Lett. 76, 647 (2002) [Pisma Zh. Eksp. Teor. Fiz. 76, 771 (2002)].

V. G. Bornyakov et al. Nucl. Phys. Proc. Suppl. 119, 739 (2003) [arXiv:hep-lat/0209029].

[14] P. Y. Boyko, F. V. Gubarev and S. M. Morozov, arXiv:0704.1203 [hep-lat].

P. Y. Boyko, F. V. Gubarev, S. M. Morozov, PoS LAT2007, 307 (2007) [arXiv:0712.0656 [hep-lat]].

[15] H. Meyer, M. Teper, JHEP 0412, 031 (2004) [arXiv:hep-lat/0411039].

B. Lucini, M. Teper and U. Wenger, JHEP 0406, 012 (2004) [arXiv:hep-lat/0404008].

B. Lucini and M. Teper, Phys. Rev. D 64, 105019 (2001) [arXiv:hep-lat/0107007].

B. Lucini and M. Teper, JHEP 0106, 050 (2001) [arXiv:hep-lat/0103027].

B. Lucini and M. Teper, Phys. Lett. B 501, 128 (2001) [arXiv:hep-lat/0012025].

[16] M. Luscher and P. Weisz, JHEP 0109, 010 (2001) [arXiv:hep-lat/0108014].

Y. Koma, M. Koma and P. Majumdar, Nucl. Phys. B 692, 209 (2004) [arXiv:hep-lat/0311016].

[17] S. Kratochvila and P. de Forcrand, Nucl. Phys. B 671, 103 (2003).

[18] H. B. Meyer, JHEP 0401, 030 (2004) [arXiv:hep-lat/0312034].

H. B. Meyer, JHEP 0301, 048 (2003) [arXiv:hep-lat/0209145].

[19] M. Luscher and U. Wolff, Nucl. Phys. B 339, 222 (1990).

[20] A. Di Giacomo, G. C. Rossi, Phys. Lett. B 100, 481 (1981).

A. Di Giacomo, G. Paffuti, Phys. Lett. B 108, 327 (1982).

M. Campostrini, A. Di Giacomo and G. Mussardo, Z. Phys. C 25, 173 (1984). 